Cahiers d'études africaines

187-188|2007

Les femmes, le droit et la justice

\title{
Genre et justice
}

Les recherches avancées en langue anglaise

\section{Catherine Coquery-Vidrovitch}

\section{OpenEdition}

\section{Journals}

Édition électronique

URL : http://journals.openedition.org/etudesafricaines/7742

DOI : 10.4000 /etudesafricaines.7742

ISSN : 1777-5353

Éditeur

Éditions de l'EHESS

Édition imprimée

Date de publication : 15 décembre 2007

Pagination : 461-494

ISBN : 978-2-7132-2140-8

ISSN : 0008-0055

\section{Référence électronique}

Catherine Coquery-Vidrovitch, « Genre et justice », Cahiers d'études africaines [En ligne], 187-188 | 2007. mis en ligne le 15 décembre 2010, consulté le 10 décembre 2020. URL : http://

journals.openedition.org/etudesafricaines/7742; DOI : https://doi.org/10.4000/etudesafricaines.7742

Ce document a été généré automatiquement le 10 décembre 2020.

(c) Cahiers d'Études africaines 


\title{
Genre et justice
}

Les recherches avancées en langue anglaise*

\author{
Catherine Coquery-Vidrovitch
}

1 Un regard sur la bibliographie existante sur le sujet démontre la richesse relative des recherches en langue anglaise, face à la pauvreté des travaux de langue française. En anglais, les travaux historiques sur les archives juridiques furent lancés par Martin Chanock (1982). Ils sont entretenus depuis lors par les tables rondes annuelles organisées à Stanford University par l'historien Richard Roberts, qui ne se lasse pas de souligner la richesse des sources juridiques en histoire africaine (Mann \& Roberts 1991), en particulier en ce qui concerne les sources francophones (Roberts 2005). Depuis peu, les ouvrages se multiplient ${ }^{1}$. En français, peu de travaux ont été effectués sinon sous forme d'articles ou de mémoires inédits (notamment à l'Université de Dakar) depuis l'ouvrage majeur du sociologue Abdoulaye Bara Diop (1985) sur la famille wolof.

2 L'histoire de la condition féminine en Afrique sous la colonisation est encore peu développée, en raison, croyait-on, de la pauvreté des sources. La toute première enquête française à se préoccuper officiellement des femmes fut lancée dans le cadre de la mission d'information dite Guernut organisée par le gouvernement du Front populaire dans l'ensemble de l'Empire ; elle se traduisit par un rapport de plus de deux cents pages rédigé par Denise Savineau en 1938 qui ne couvre d'ailleurs pas le Sénégal (Lydon 1997 ; Barthélémy \& Savineau 2001). Jusqu'à présent, ce que l'on sait du droit des femmes a surtout été rassemblé, cercle par cercle et région par région, dans les Grands Coutumiers de l'AOF, trois volumes publiés en 1938 à partir des nombreuses enquêtes demandées depuis le début de la décennie aux administrateurs locaux, chargés de recueillir auprès des chefs tout ce qu'ils pouvaient sur les « coutumes » de leur région. Ces textes marquent le démarrage de l'intérêt porté à la place des femmes dans la société. Car nombreux furent les renseignements glanés à cette occasion sur tout ce qui pouvait constituer un objet de litiges concernant les femmes: usages et modalités des mariages, des divorces, des héritages, etc., qui s'avèrent extrêmement divers selon les régions et les groupes sociaux alors dits « ethniques », voire «tribaux ». Du côté anglophone, un usage similaire fut fait des décisions de jurisprudence collectées au fil du temps. On doit souligner le caractère reconstruit de ces données 
supposées "traditionnelles", dans la mesure où elles cumulaient deux sources de préjugés : d'une part, évidemment, ceux des administrateurs pénétrés du droit romain de la primauté du seul chef de famille mâle, et qui en outre comprenaient plus ou moins bien, et interprétaient de même ce qu'il leur était conté ; et, d'autre part, celui des chefs, la plupart du temps de grands anciens protégés de l'administration coloniale ; les chefs étaient souvent imbus de leur autorité d'un type relativement nouveau, et soucieux de transmettre une loi rigidifiée tout à leur avantage, celui de leurs souhaits autant que des réalités. En particulier, à propos des femmes, cette source exclusivement masculine et souvent rétrograde a eu tendance à durcir le poids de préjugés visant à " rétablir » la condition féminine dans des limites supposées héritées, donc hostiles à toute possibilité d'émancipation des règles patriarcales usuellement dominantes. Ajoutons que les Grands Coutumiers, à la différence des recueils de jurisprudence rassemblés dans les colonies britanniques, n'avaient pas force de loi auprès des tribunaux même "indigènes"; ils ne servaient tout au plus que de référence informative.

3 Il est donc vrai que, jusqu'à il y a peu, nous savons peu de choses, a priori, sur la condition des femmes et sur leurs réactions ordinaires qui n'intéressaient guère le colonisateur : celui-ci recrutait, pour ses différents objectifs, une main-d'œuvre quasi exclusivement masculine, y compris pour le travail domestique. C'est pourquoi les sources juridiques sont particulièrement utiles : nous n'entendons parler des femmes que lorsque celles-ci sont intervenues en justice. Or les conflits furent nombreux, pour deux raisons apparemment contradictoires. La première est que, comme les usages britanniques, le droit français hérité du code Napoléon était parfois à sa façon plus défavorable aux femmes que le droit coutumier ${ }^{2}$; notamment en pays de tradition matrilinéaire où la progéniture d'un couple relevait du lignage maternel et non de celui du père, ou bien où les gains de la femme n'appartenaient pas au mari, les conflits se multiplièrent; d'une façon générale, le jeu entre la loi française et les usages coutumiers pouvait intervenir en faveur de l'un ou l'autre plaignant. La seconde raison est que, pour se défendre, la femme, qui avait en règle générale peu de chances de se faire entendre au niveau coutumier, eut vite fait de comprendre néanmoins que le tribunal colonial lui donnait peut-être plus de chance. Dès qu'elles le purent - surtout en ville - elles portèrent plainte.

4 L'inconvénient est évidemment que les sources juridiques n'interviennent qu'en cas de conflit : on ne connaît pas les innombrables situations où le tribunal ne fut pas invoqué. Il y a donc risque, d'une part d'exagérer la capacité de recours des femmes à la justice et, d'autre part, de souligner excessivement les situations de crise par rapport à la réalité. Cela dit, les modalités de la justice, et l'évolution à travers le temps de la jurisprudence qui, à partir de la même loi, put en donner à cinquante ans d'intervalle une interprétation très différente, sont instructives de la condition féminine et de son évolution à travers la période coloniale.

5 Ces conflits entre hommes et femmes, entre droit dit coutumier et droit colonial, entre droits du lignage et droits individuels, intervinrent d'abord dans des affaires de sorcellerie, puis surtout dans deux domaines privilégiés du droit civil : celui du mariage (et surtout du divorce), et celui des droits de propriété (et de leur transmission). C'est donc sur des exemples de ce type que nous nous arrêterons, à partir de travaux exemplaires effectués sous la colonisation britannique, qui ne peuvent qu'inspirer des travaux analogues souvent encore à innover sous la colonisation française. 
Une périodisation des rapports de genre?

Revenons d'abord sur ce fait établi par Martin Chanock (1982) : contrairement à ce qui est souvent supposé, il n'y a pas eu deux droits successifs et conjoints en Afrique : le «traditionnel » et le «moderne » - à savoir, pour ce dernier, occidental de tradition britannique ou française suivant les cas. Il y en eut au moins trois : un droit ancien travaillé aux temps précoloniaux, c'est-à-dire lui-même en évolution, car ces temps furent millénaires et chahutés. De ce droit ancien, variable dans l'espace aussi bien que dans le temps, nous ne connaissons que des bribes, tel qu'il fut parfois évoqué par les premiers voyageurs qui ont $\mathrm{pu}$ nous rapporter des «traditions» constamment remaniées, voire manipulées par les générations successives.

7 Ce que nous connaissons bien, en revanche, c'est le «droit coutumier " colonial, tel qu'il fut en partie refabriqué par l'alliance entre les pouvoirs anciens laissés en place et l'intervention souvent musclée du colonisateur. C'est ici qu'il faut connaître les analyses percutantes de Martin Chanock, qui fut le premier à en déduire une périodisation. Dans la dernière phase précoloniale, au $\mathrm{XIX}^{\mathrm{e}}$ siècle, la condition des femmes eut à souffrir d'un contexte agité où les guerres de conquête, l'accroissement de l'esclavage interne et la réduction de leurs droits économiques allèrent de pair. Les rapports de genre devinrent encore plus inégaux qu'auparavant. En revanche, ensuite, au début de l'ère coloniale, la juxtaposition et la coexistence des différentes strates de droit semblent avoir brièvement doté les femmes d'une relative liberté de jeu, car certaines d'entre elles furent rapides à comprendre qu'elles pouvaient arguer de ces contradictions à leur avantage, notamment dans le domaine du mariage et de la résidence. Ainsi, dans le district de Goromonzi proche de la capitale (Rhodésie du Sud occidentale), sur les 345 plaintes civiles instruites par le Native Commissioner, entre octobre 1899 et février 1905, 95 concernaient des jeunes filles qui refusaient d'épouser un homme qui avait déjà payé la dot, et 65 impliquaient des épouses fugitives. La moitié des plaintes provenaient de maris qui réclamaient le retour d'une épouse récalcitrante (Schmidt 1991 : n. 38). Mais, bientôt, le machisme ambiant reprit le dessus : car cette brève période provoqua une réaction de conservatisme social de la part des aînés dès qu'ils furent invités à participer à une parcelle du pouvoir colonial. Ils voyaient avec inquiétude se dissoudre leur emprise sur les jeunes et sur les femmes. Cette réaction leur permit de rejoindre le conservatisme des administrateurs coloniaux dans la confection d'un «droit coutumier» qui fit écho à leur commune préoccupation de contrôle des femmes.

8 En fait, le travail juridique fut complexe car, de leur côté, les juristes occidentaux étaient mal préparés à comprendre les nuances du droit local. Chanock étudie dans le détail les modalités de la fixation du droit "coutumier » en matière de lévirat en Rhodésie du Nord (Zambie) dans les années 1920. Avant que les cours de justice britanniques n'aient édicté qu'une femme ne pouvait être forcée au mariage, le lévirat était couramment appliqué dans la région de Boma. En vertu de la compensation matrimoniale que le lignage du mort avait versée à la famille de son épouse, celle-ci devait rester et accepter le remariage avec un frère, voire un fils de son défunt mari. Or, de plus en plus de femmes refusaient d'être ainsi liées à l'héritier, d'où les recours en justice, et la nécessité pour la cour de Boma de " fixer la coutume ». Elle entreprit de le faire en posant aux chefs locaux une série de questions précises auxquelles il fut répondu de façon nuancée, nuances que les magistrats n'entendirent pas. Ainsi, il leur fut confirmé qu'en théorie la femme qui refusait d'être héritée devait restituer la dot 
mais, dit un témoin, « d'autres pouvaient ne pas la réclamer »; si la famille de la femme entretenait avec celle du mari des relations amicales, « l'héritier attendrait et verrait». Autrement dit, il y avait la règle, mais aussi des adaptations et des arrangements possibles qui font que, dans l'ancien temps, la règle n'avait rien d'absolu. Or cette rigidité fut dès lors affirmée : ou bien restituer la dot, ou bien rester...

Restituer la dot introduisait une nouveauté quasi impensable auparavant : la possibilité pour une femme de divorcer. L'usage, dans la région, était que les femmes fussent mariées à vie, et certainement pas qu'elles aient le droit de décider de s'en aller. Certes, certaines le faisaient déjà, mais justement ! Il n'était pas question d'exiger de force la restitution de la dot, ce qui aurait rendu la rupture irrévocable. Tout reposait donc sur l'attente, la discussion, l'arrangement, la patience, bref la palabre. Ce processus fut bel et bien supprimé par l'intervention d'un droit écrit rigoureux (Chanock 1982 : 57-59). De même, dans les années 1930, les maris de Rhodésie du Sud réclamaient désormais le divorce (et, bien entendu, la restitution de la dot) : des 171 cas entendus entre juillet 1931 et juillet 1939 dans le district de Goromonzi, 128 (soit 75 \%) étaient des demandes de divorce émanant du mari, contre seulement 14 qui réclamaient encore le retour de la fugitive (Schmidt 1991). Ainsi le droit coutumier colonial généra-t-il un droit nouveau qui, en ce cas précis comme en beaucoup d'autres, n'avait plus grand-chose à voir avec les usages anciens.

10 Le droit colonial fut, nous l'avons dit, rétrograde vis-à-vis des femmes, et ce jusqu'au milieu du $\mathrm{xx}^{\mathrm{e}}$ siècle. L'attitude face à l'adultère en offre un exemple criant. Là encore, Chanock propose pour la Rhodésie du Nord une analyse éclairante. Il s'agit d'une zone d'Afrique où les femmes, esclaves ou non, étaient clairement devenues au début de la colonisation - si elles ne l'étaient auparavant - un objet d'échange matrimonial qui n'avait guère voix au chapitre. Entre autres, deux procès en adultère furent présentés à la cour de Boma dans les années 1913-1916. À nouveau, celle-ci entreprit, par enquêtes, de fixer le droit coutumier. Les témoignages concordent pour distinguer le cas d'épouses de chefs ou de simples hommes libres. Dans le premier cas, il fut affirmé que le châtiment du coupable - c'est-à-dire l'homme trompant le mari - était sévère, l'éventualité étant soit la mort, soit la mise en esclavage, soit une très forte amende ; seule cette dernière était requise s'il s'agissait d'un homme du commun. En tout état de cause, on remarque l'absence de châtiment à l'égard de la femme, considérée comme irresponsable. Cet héritage juridique heurta la conscience des magistrats britanniques pour deux raisons : à leurs yeux, pénétrés comme ils l'étaient de morale chrétienne, la coupable était la femme qui avait commis une faute morale ; l'adultère relevait du droit privé et non d'une affaire criminelle. D'où de nombreuses discussions avec les responsables africains. Les administrateurs admirent un moment, entre 1916 et 1933, que, d'après la loi coutumière, l'adultère relevait de la justice criminelle - sans aller néanmoins jusqu'à la mort du coupable. Ils suivaient en ceci l'argumentation des chefs qui alléguaient (comme de leur côté les Britanniques) une tendance à la dissolution des mœurs, et qui en profitèrent donc pour affirmer outrageusement leur pouvoir et leurs privilèges. Or, rien n'est moins sûr : il ne vint pas à l'esprit des juges, par exemple, que si on leur rapportait que tel ou tel avait tué un coupable récidiviste, ce n'était pas nécessairement par acte de justice mais tout bonnement par vengeance... L'autre pierre d'achoppement était la notion de faute qui, pour les Britanniques, était non celle de l'homme mais de la femme coupable d'adultère. Les juges étaient incapables de comprendre la logique qui faisait qu'après avoir reçu compensation le mari n'envisageât pas de renvoyer la femme, puisqu'elle lui appartenait. Il leur paraissait 
immoral qu'en définitive le mari tirât un avantage financier du commerce de son épouse avec un autre (Chanock 1982 : 59-63). Sous l'influence du christianisme, on voit progressivement les Africains adopter une position analogue: au nom de leurs « coutumes », ils ont préféré intérioriser la règle d'une amende également imposée à la femme plutôt que le risque, impensable pour eux, d'avoir à la renvoyer à sa famille, ce qui aurait posé le problème de la restitution de la dot.

11 L'évolution fut analogue en Rhodésie du Sud, où la Native Adultery Punishment Ordinance, ratifiée dès 1916, fit de l'adultère d'un Africain avec une femme mariée une offense criminelle passible pour les deux partenaires d'une amende de $100 £$ ou d'une peine d'un an de prison avec travail forcé. Forts de l'appui britannique, les chefs locaux allèrent encore plus loin dans les années 1930 : insistant sur la licence accrue des femmes, ils réclamèrent l'introduction de châtiments corporels contre les épouses passibles d'adultère ou de désertion, et contre les filles qui fuyaient en ville contre l'avis de leurs parents. En 1933, le Native Commissioner de Salisbury proposa même d'exiger des femmes en ville - ce qui se faisait déjà dans les «centres extracoutumiers » belges - un passe qui prouverait leur statut de femme mariée ou sous tutelle (Schmidt 1991: 739, 755-756). La justification du châtiment répondait au sens britannique aigu de la propriété privée : puisque l'homme payait la dot, la femme était son bien. Un commis des "affaires indigènes » l'explicitait en 1914: "l'adultère arguait-il - est une atteinte sérieuse au droit de propriété, comparable au pire des vols $»^{3}$. L'administration coloniale s'inquiétait pour sa part de voir les femmes utiliser l'ordonnance de 1916 pour affirmer leur liberté de choix "as a cloak for licence" (" pour déguiser leur dépravation»), chaque fois qu'elles avaient envie de changer de mari ${ }^{4}$. Le droit coutumier colonial était donc, non seulement en grande partie nouveau, mais fut aussi évolutif. D'où l'intérêt d'étudier en ce domaine la transformation des jurisprudences.

12 Si l'on se tourne vers l'ancienne AOF, on constate que c'est seulement après la Seconde Guerre mondiale qu'on associa quelques femmes privilégiées aux droits politiques (le droit de vote) accordés à un corps électoral «indigène » censitaire par la «France d'outre-mer» créée en 1946. Paradoxalement, en ce qui concerne l'Afrique exfrançaise, les droits politiques furent ensuite élargis plus rapidement pour les femmes au suffrage quasi universel, puisque le droit de vote fut accordé en 1952 à toutes les femmes majeures ( 21 ans à l'époque) mères de deux enfants - ce qui était sauf accident le cas de la quasi-totalité d'entre elles (Coquery-Vidrovitch 1994: chap.17). En revanche, l'égalité civile n'est encore aujourd'hui, en règle générale, pas reconnue aux femmes, en particulier en ce qui concerne le mariage. Ainsi, au Sénégal, la polygamie demeure-t-elle légale à condition d'être prévue explicitement dans le contrat de mariage. En Tanzanie, l'un des pays les plus avancés en ce qui concerne l'émancipation des femmes, celle- ci demeure définie et limitée de façon assez vague, comme « le droit de divorcer, le droit de consentir au mariage, le droit d'être reconnues dans la société, le droit de posséder et d'hériter un bien, et le droit à la sécurité dans le mariage $»^{5}$.

Femmes et sorcellerie

13 C'est un domaine sur lequel nous n'insisterons pas, n'étant pas spécialiste. Mais c'est un des terrains par excellence où les sources judiciaires peuvent être d'un grand apport, précisément parce que les accusations de sorcellerie ont amené à exercer sur les femmes des sévices (ordalies, empoisonnements, etc.) qui ont assez souvent conduit leurs auteurs devant la justice coloniale. On s'aperçoit d'ailleurs, a contrario, que les 
femmes, souvent impliquées dans les affaires de sorcellerie, ont aussi su parfois tirer leur épingle du jeu. Ainsi les registres d'un magistrat isolé mais consciencieux, en poste aux confins de la Tanzanie et de la Zambie actuelles entre 1897 et 1903 (Wright 1982), révèlent déjà la fréquence du processus; le magistrat note en 1900 que, bien que proclamée illégale, "la coutume de boire le mwavi [une mixture empoisonnée pour déterminer s'il y a eu faute ou acte de sorcellerie] est si répandue que les sentences les plus sévères ne parviennent pas à l'éradiquer ». Deux au moins des affaires qu'il eut à traiter révèlent le rôle actif des femmes. Dans le premier cas, un dénommé Chitembwa, qui finit par succomber à l'ordalie, avait battu sa femme dans un accès d'ivresse. Celleci était morte peu après. Sa mère, à l'occasion d'une cérémonie de divination, accusa le gendre d'être le coupable, et c'est celui-ci qui, de lui-même, avait réclamé au nganga (docteur ou sorcier) de lui administrer le mwavi dont il mourut...

14 Aux temps précoloniaux, la coutume, dans la région, aurait voulu que seul le chef autorisât l'ordalie par le poison. Mais, compte tenu de l'interdiction coloniale, les chefs évitaient soigneusement de se compromettre dans une telle affaire. Quand Fwambo, le chef de Mambwe, découvrit aux environs de son enclos un homme en train de cacher une substance douteuse, il le fit appréhender et c'est à ses femmes qu'il le confia. Celles-ci le battirent puis le forcèrent à goûter la mixture : lui aussi en mourut... Le cas révéla qu'il s'agissait d'un étranger, précédemment chassé de sa communauté pour fait de sorcellerie, reconnu coupable d'avoir trahi la confiance de son nouveau protecteur dont les femmes étaient chargées de faire respecter le domaine. Ainsi, dans les deux cas, les femmes étaient impliquées dans la manipulation par le poison. Néanmoins, la plupart des autres affaires démontrent au contraire comment les chefs usaient et abusaient de l'épreuve par le poison pour détecter parmi leurs nombreuses femmes, signes de leur richesse, les "sorcières ». Au Malawi, les cas de châtiments infligés aux supposées sorcières dans l'Entre-deux-guerres furent étudiés de façon systématique à partir des archives juridiques dans un article au titre évocateur : "European Courts Protect Women and Witches: Colonial Law Courts as Redistribution of Power in Swaziland 1920-1950» (Booth 1992).

15 Geschiere (1995 : chap. 5), qui a étudié l'évolution de la sorcellerie au Cameroun depuis l'indépendance, remarque que la justice s'en mêle de plus en plus, mais que les cas concernent peu les femmes: peut-être parce qu'il n'y a procès que lorsqu'il s'agit d'hommes importants, tandis que la sorcellerie féminine demeure au niveau populaire. Sur un échantillon local en pays maka de 38 cas, il ne compte que six femmes, qui pourtant dans les villages étaient réputées être au moins aussi actives que les hommes en sorcellerie. Ce qui est sûr, c'est que l'État intervient de plus en plus en ce domaine. L'analyse des procès, malgré les diatribes des fonctionnaires qui dénoncent, dans la sorcellerie des villageois, une forme de subversion permanente, met plutôt en scène des personnages plus ou moins "modernes» à l'intérieur du village -instituteurs, planteurs aisés, cadres du parti, innovateurs économiques - qui apparemment se sentent davantage menacés parce qu'ils craignent la jalousie de leurs voisins : le cas est classique du riche planteur qui est envoûté par son voisin. Le rôle-clé est joué par les guérisseurs qui fournissent les "preuves » contre les sorciers.

Les ordalies ont connu, comme les Églises noires, une forte recrudescence dans les moments de crise coloniale. Ce fut le cas en Casamance pour laquelle il serait utile de faire des recherches à ce propos dans les procès de l'époque. Ce fut aussi le cas en 
Angola à l'occasion des troubles sur le front oriental des opérations du MPLA (Mouvement populaire de libération de l'Angola) entre 1966 et 1975 (Brinkman 2003).

Le statut du mariage et son évolution à travers les textes juridiquesDroit hérité et droit « moderne»

L'interprétation des documents juridiques de première main s'avère précieuse pour élucider l'évolution éventuelle des stratégies nuptiales des femmes. La recherche n'avait été qu'approchée du côté francophone où, à l'exception d'ouvrages plus anciens d'ethnologie qui ont acquis le statut de source d'histoire, le seul travail sérieux sur la question fut la thèse de sociologie sur La famille wolof citée supra, auquel on peut évidemment ajouter quelques travaux de qualité, souvent en anglais, car les Britanniques ont exploré la question depuis un moment déjà ${ }^{6}$.

Ainsi, les registres du magistrat déjà cité révèlent à quel point la mise en gage ou en esclavage des fillettes et des femmes était devenue en Afrique centro-méridionale une pratique courante au tournant $\mathrm{du} \mathrm{xx}^{\mathrm{e}}$ siècle (Wright 1982). Pour celles-ci, se réfugier dans le petit centre administratif nouvellement créé était devenu le seul recours. En 1899, deux femmes du chef Chungu avaient fui et demandé l'aide du magistrat à Abercorn, arguant qu'elles avaient été trompées et quasiment chassées; le chef de son côté réclamait une compensation égale à la dot qu'il avait versée. Le magistrat autorisa les femmes à rester avec les hommes de leur choix, à condition que chacun d'eux payât cinq beaux pagnes à Chungu.

Le cas fut plus délicat en 1901 : les femmes du chef Changala s'étaient réfugiées avec leurs nouveaux maris à la mission des Pères Blancs de Kayambi ; quant aux épouses du chef Ponde, elles avaient fui dans le district voisin pour vivre avec les hommes de leur choix. Le magistrat demanda instamment au père supérieur de lui renvoyer les femmes à Abercorn, afin qu'il réglât lui-même le différend avec le chef; sans aucun doute, quand il s'agissait d'hommes importants, le magistrat cherchait à jouer de son autorité juridico-politique pour amadouer le chef tout en remédiant à la pratique locale de gager les femmes pour s'acquitter d'une faute - que ce soit une dette, une offense ou plus précisément un adultère. Des cas dont il eut à traiter ressort la variété des mobiles qui poussaient les femmes à la fuite : plusieurs avaient quitté leur mari juste parce que le salaire urbain d'un autre prétendant leur paraissait de meilleur augure. De plus en plus, aller à la ville leur permettait de se marier sans attendre de la famille du prétendant le paiement d'une compensation matrimoniale qui, de surcroît, les eût engagées ailleurs envers un vieux mari qui ne leur convenait guère : trop âgé, grand polygame, traitant d'esclaves... La plupart du temps, la plainte qui remontait jusqu'au magistrat avait à faire avec une forme ou une autre de mauvais traitement: épouse battue, veuve obligée d'accepter contre son gré le frère de son mari (coutume du lévirat), ou tout bonnement surexploitation de l'outil féminin de production ; ainsi des femmes achetées comme épouses-esclaves cherchaient à obtenir de l'autorité britannique leur liberté, ou plus simplement leur droit à disposer du salaire de leur travail jusqu'alors payé au mari qui les louait. On note aussi le cas du frère d'un homme décédé qui, au nom du lévirat, avait loué le travail de sa nièce encore fillette à la mission : le magistrat le condamna à rendre l'enfant à sa mère. Lui revenait aussi le cas de femmes violées par des auxiliaires coloniaux de passage dans des villages isolés; pendant longtemps, la confusion fut entretenue dans la région entre l'hospitalité à l'ancienne, qui faisait participer à l'offrande au voyageur une fille de la maison, et le 
pillage par des bandes de soudards... Avec le magistrat d'Abercorn, les agents usant de leur charge officielle pour des abus de ce genre furent sévèrement traités.

Enfin, en 1910 encore, on signale dans la même région un cas qui traduit bien le jeu possible entre la loi dite coutumière et la loi britannique; il s'agit d'un homme déjà pourvu d'une épouse qui, venu s'expatrier dans la région voisine, avait pris localement femme dont il avait eu un enfant. Retourné dans sa région d'origine, il se convertit au protestantisme et se maria religieusement puis revendiqua, soutenu par les missionnaires, son droit patrilinéaire à récupérer l'enfant de sa précédente union. Dans un texte assez long où le magistrat explique ne se référer que de façon limitée à la loi anglaise (qui, ne reconnaissant aucun droit à la mère, se fiait exclusivement dans ce genre de cas à l'intérêt de l'enfant) ou au droit coutumier (qui ne connaissait que le droit du patrilignage), il décide de repousser la revendication des missionnaires et de restituer l'enfant à la mère, au motif qu'elle avait dûment et longuement été abandonnée par le plaignant. Bref, comme le constatait à propos d'adultère, en 1916, un chef zambien désabusé :

«Les femmes devraient être punies. Mais quand nous les punissons, elles courent se plaindre chez le magistrat » (Chanock 1982 : 63).

21 Ce qui a été observé en ce coin perdu d'Afrique a dû se reproduire souvent ailleurs. E. Schmidt (1991 : 735) décrit pratiquement la même évolution en Rhodésie du Sud entre les deux guerres. Un cas précoce fut celui de femmes qui fuyaient le mariage pour poursuivre leurs études: c'est ce qu'il advint en Rhodésie du Sud pour Emelda Madamombe (née en 1918), qui se réfugia à la mission pour échapper au mariage prévu par son frère en échange d'une forte dot. La volonté de devenir religieuse (" brides of Christ ») à la mission catholique fut aussi fréquemment invoquée entre les deux guerres par des filles qui fuyaient un mariage arrangé ou le lévirat, quitte à utiliser leur salaire pour dédommager le tuteur de la dot qui sinon lui échappait (ibid. : 749-750). On connaît aussi, par les interviews de vieilles prostituées, les raisons de leur arrivée, adolescentes, à Nairobi au début de ce siècle : le plus souvent, c'était parce qu'elles refusaient de se laisser marier par leur famille à des hommes qui, pour une raison ou une autre, les rebutaient ; parfois aussi, c'était pour suivre un amoureux en ville (Bujra 1975).

Cela dit, il ne faudrait pas se limiter à un dualisme simpliste: d'un côté les femmes opprimées par un droit rétrograde, et de l'autre les femmes rebelles qui utilisent tous les recours de la loi coloniale pour échapper au carcan coutumier. L'étude du mariage chez les femmes gusii du Kenya montre que les stratégies féminines furent plus complexes. C'est une région où, après les années 1940, en raison de l'ouverture de la région à l'économie de marché, le prix de la dot connut une inflation considérable. Il devenait de plus en plus difficile pour un jeune homme d'acquérir une épouse, tandis que les familles cherchaient à imposer à leurs filles des hommes riches donc âgés et polygames. Dans les années 1950, la plupart des hommes de famille pauvre ne pouvaient pas rêver de se marier avant la quarantaine passée, et beaucoup étaient condamnés à rester célibataires. Un des chefs réussit à limiter les dégâts en fixant la dot à six vaches et un taureau, mais les vieux pratiquèrent la surenchère. Or les mœurs modernes firent aussi leur entrée, et les filles se mirent à défendre devant les cours de justice leur droit à choisir un plus jeune époux : des centaines de cas furent soumis aux cours locales dès avant l'indépendance. Parmi les 900 procès criminels étudiés, on compte 685 cas d'adultère et 215 enlèvements de femmes; les 380 procès civils concernent 160 divorces et 220 "récupérations» d'épouses (Shadle 2006). Le tout 
représentait plus de $20 \%$ des procès. L'ensemble démontre que, si les jugements tenaient grand compte de l'avis des aînés, ils ne furent pas toujours sourds aux revendications des hommes jeunes ni des femmes, notamment si celles-ci étaient exagérément battues ou maltraitées par le mari déserté, et surtout si elles pouvaient arguer de témoins convaincants parmi les membres de leur propre famille. L'épouse plaignante ou accusée n'hésitait pas à accuser le mari abandonné de brutalité ou de stérilité, et à vanter les mérites de l'époux de leur choix, voire l'amour qu'elle éprouvait pour lui. Au grand dam de l'accusateur qui récusait pour les femmes le droit au consentement, elles allaient jusqu'à revendiquer explicitement la responsabilité de leur choix. En revanche, même dans le cas d'enlèvement, elles tenaient à régulariser leur nouvelle union. Elles ne remettaient donc aucunement en cause l'usage coutumier de la compensation matrimoniale. La plupart du temps, les couples ne s'enfuyaient dans la province voisine que le temps de négocier son paiement: la question ne concernait d'ailleurs que les hommes, car seul l'homme était poursuivi en cas d'adultère. Sauf si le mari exigeait le retour de sa femme, auquel cas les aînés lui donnaient généralement raison, le recours à la justice servait à fixer le règlement de la dot (qui assurait au moins en partie le dédommagement du mari ou de la famille de la femme) et permettait aux épouses illégitimes de réintégrer leur cadre social coutumier : de tous les cas étudiés, un seul concernait une prostituée qui revendiqua ouvertement sa volonté de vivre seule en dehors du mariage.

En conclusion, la recommandation faite en fin d'article par Marcia Wright dès 1982 reste d'actualité : la nécessité de se mettre en quête de sources juridiques toujours plus nombreuses, de façon à mieux comprendre le contexte des bouleversements sociaux et politiques de telles affaires.

Mariage et divorce en ville

En ville, l'«élite» urbaine embourgeoisée a évolué en fonction du contexte et de la religion. L'islam pose problème, dans la mesure où mariage et répudiation étant longtemps restés, et demeurant souvent encore une affaire strictement privée, les actes de justice sont relativement rares sur la question. Il n'empêche que la situation a, là encore, été assez bien étudiée dans la littérature anglo-saxonne. Citons le cas des îles de Lamu (Kenya) quasi exclusivement musulmanes où Patricia Romero Curtin (1984) a démontré à quel point le divorce est entré dans les mœurs, ou celui de Zanzibar pour lequel la question de savoir ce qu'est au juste un divorce agita fort la cour de justice (Styles 2003). C'est que, comme le remarque Richard Roberts (2005) à propos du Soudan occidental, à travers le droit les femmes recherchent le divorce alors que les hommes recherchent le pouvoir.

Dans les régions de culture swahili, l'islam n'a pas réduit le nombre de divorces, au contraire, puisque la répudiation par l'homme est facile là où elle s'exerce à plein (Le Guennec-Coppens 1987). Le divorce atteint à Lamu une sorte de niveau record, sans que la question soit vraiment traitée par la justice : le taux en a été, entre 1969 et 1978, de $73 \%$ par an ; plus de la moitié des femmes qui se sont mariées durant cette période en étaient à une deuxième, voire une quatrième union. Tout se passe comme si le premier mariage était une sorte de rite de passage obligé, l'initiation à la vie adulte par une union arrangée par les parents. La femme est très jeune, à peine nubile, de façon à éviter qu'elle ait auparavant perdu sa virginité. Parmi les familles les plus anciennes, où le rang social est élevé et la polygamie importante puisque les hommes en ont les moyens, les divorces sont moins nombreux car ils remettraient en cause trop 
d'alliances d'intérêts et de convenances. Mais dans les milieux populaires, les femmes vivent dans la plus grande insécurité : « Un mari est seulement un ami... il n'est qu'une porte de natte ", disent deux proverbes swahili ; autrement dit, il est une assurance des plus précaires. Le moindre manquement de la femme, la stérilité bien sûr, un enfant anormal ou un soupçon d'infidélité suffisent pour que l'homme prononce la formule sacramentelle, devant témoin ou non. La femme, en revanche, doit arguer de motifs graves: dans seulement $5 \%$ des cas, ce sont elles qui ont pris l'initiative devant la justice. La plupart n'ont pas le choix ; pour obtenir le divorce, elles doivent se mettre dans une situation qui incite le mari à recourir à la répudiation : mauvais caractère, refus de le nourrir et, bien sûr, adultère. Ce recours privilégié à la loi coranique (sans l'intervention, comme au Sénégal, de tribunaux musulmans dûment contrôlés par l'administration) peut expliquer la rareté relative des études sur le mariage en pays d'islam : tant que les époux n'ont pas recours aux lois civiles «modernes », les sources juridiques restent rares, sinon inexistantes.

$\mathrm{Au}$ Kenya, où domine souvent une dure loi patrilinéaire pour les femmes, le gouvernement britannique a essayé de les protéger en 1959 par la loi dite Affiliation Act qui, à l'imitation de la loi votée en Grande- Bretagne deux ans auparavant, visait à protéger la femme et son enfant illégitime. L'initiative, qui cherchait à inclure la responsabilité du père, souleva tant de protestations locales qu'elle fut abrogée par le gouvernement indépendant dix ans plus tard (1969) (Thomas 2001). La mesure consistait à confier l'enfant à la mère moyennant une pension mensuelle jusqu'à 16 ans, pension qui pouvait s'élever jusqu'à 200 shillings kenyans (environ $\$ 28$ en 1959). L'opposition parlementaire et générale fut virulente, arguant que cette coutume étrangère faisait des hommes les « esclaves » des femmes. Cette campagne qui fit couler beaucoup d'encre est aisée à suivre, notamment à travers les procès qui en donnèrent l'occasion. Le débat pose la question de savoir qui devait contrôler la sexualité des femmes, et quelles responsabilités leur fécondité mettait en jeu. Il devint un enjeu politique, entre ceux qui posaient l'émancipation des femmes comme un aspect de la «modernité », et ceux qui revendiquaient au contraire le respect des «traditions » au sein de l'État devenu national. Il révèle la perméabilité entre les notions apparemment contradictoires de société «civile» ou " coutumière ». La presse posa par exemple la question de savoir s'il était possible de poursuivre deux fois le même homme, l'une en vertu de la loi, et l'autre en raison de la compensation traditionnellement due à la naissance d'un enfant: celle-ci était déjà prévue par la loi coutumière coloniale qui reconnaissait au père de la femme le droit de poursuivre l'homme au cas où celui-ci refuserait d'épouser sa fille. Or la nouvelle loi tentait de donner à la femme considérée par ses opposants comme une prostituée - un droit réservé jusqu'alors aux hommes de la famille. C'est la raison pour laquelle les administrateurs - assimilant les ruraux aux tenants de la «tradition »- recommandèrent de n'appliquer la nouvelle loi qu'en milieu urbain.

La procédure constitue évidemment pour l'historien une source précieuse d'indices sociaux (par exemple, 240 plaintes furent déposées en 1963), d'autant plus prometteuse que dans la plupart des autres pays anglophones (Botswana, Ghana et Zimbabwe notamment) une loi analogue est toujours en vigueur aujourd'hui. La femme devait d'abord remettre un questionnaire à la cour, qui la convoquait si la plainte était jugée recevable pour une audience où elle exposait précisément les circonstances de la conception de l'enfant; si la cour les jugeait suffisantes, l'homme était convoqué à son tour. Si un certain nombre d'entre eux firent défaut, la plupart se présentèrent, 
arguant en général pour se défendre que la femme refusait le mariage, ou bien qu'ils étaient sans ressources; il revenait alors aux juges de déterminer le montant de la pension alimentaire... Mais quand l'enfant était plus grand, fréquent était le cas où le père réclamait la garde, l'enfant devenant à cet âge une source possible de revenu. Les adversaires de la loi arguaient d'ailleurs qu'elle servait surtout l'intérêt des parents de la fille utilisée comme un appât. Finalement, la loi fut abrogée pour deux raisons : la première, parce que le scandale commençait à éclabousser certains membres du Parlement (entièrement masculin) accusés d'abuser de leur pouvoir pour séduire des fillettes ; la seconde, parce que, en année d'élection, le parti dominant, le KANU (Kenya African National Union) de Kenyatta entendait apparaître comme le champion de l'idéal nationaliste face à toute mesure considérée comme une "loi étrangère " imposée par le colonisateur, et de ce fait susceptible de corrompre les mœurs en favorisant l'immoralité sexuelle et la prostitution. Bref, l'analyse de l'appareil et des arguments juridiques entourant la mesure renseigne sur le poids des usages et des contraintes sociales et culturelles pesant sur la condition féminine contemporaine au Kenya. Celle-ci reste dure : plusieurs tentatives pour faire voter au Parlement une loi interdisant au mari de battre sa femme ont échoué. La féministe radicale Chelegat, qui en proposa une fin 1978, se fit répliquer par un autre parlementaire que «cela était tout à fait africain d'enseigner aux femmes les bonnes manières en les battant ». En 1986, faute de mieux, les autobus de la capitale affichaient un panneau publicitaire: «Ne battez pas votre femme, c'est illégal. » Fin 2000, l'Attorney général proposait encore sans succès que les hommes convaincus de violence domestique fussent condamnés à un an de prison... Tous les États n'en sont pas à ce point : au Zimbabwe la loi criminalise le viol, en particulier quand l'agresseur est infecté par le sida. Mais elle n'est pas respectée ${ }^{7}$.

Dans les ports d'Afrique occidentale où la christianisation des couples « créolisés » l'a parfois emporté depuis longtemps, le statut du mariage a été largement étudié par nombre de chercheurs: ainsi à Freetown au Sierra-Leone (Harrell-Bond 1975) ou à Lagos au Nigeria (Mann 1985, 2007), ou bien à Abeokuta (Byfield 1996) ou encore en milieu " animiste » matrilinéaire à Accra au Ghana (Mikell 1991, 1995). Les actes d'état civil - mariage et divorce au premier chef -, et peut-être surtout les actes notariés à travers lesquels se décomptent les inventaires après décès et les règlements d'héritage comptent parmi les sources les plus instructives. À ce niveau, on s'aperçoit que, progressivement, les populations urbaines s'appliquèrent à se démarquer des lois occidentales, même si, à travers les archives judiciaires, Gwendolyn Mikell repère quelques cas savoureux.

C'est peut-être au fond des campagnes que l'évolution est la plus intéressante à suivre. On y constate, en particulier, que le relatif no man's land juridique des débuts du régime colonial, évoqué ci-dessus à propos d'Abercorn, souvent favorable aux femmes, prit fin avec l'interprétation «victorienne » du droit local. On y saisit bien comment la conception même du mariage reposait sur des présupposés différents. Les administrateurs coloniaux furent désarçonnés par les coutumes locales d'unions, qu'ils s'obstinaient à n'analyser que d'après leur propre conception du mariage vu comme un sacrement. C'était donc, aux yeux du colonisateur, une institution-clé, fondement des règles selon lesquelles les peuples régulaient, d'une part leur sexualité en fonction de leurs devoirs de reproduction et, d'autre part, la jouissance de leurs propriétés selon des règles d'héritage dictées par la loi du sang et de la paternité. Or, les concepts du mariage étaient, en Afrique, fondés sur de tout autres bases : ce qui était fondamental, 
ce n'était pas le lien du mariage entre deux individus, c'était l'échange assuré entre familles (naguère lignages) par la circulation des femmes et de la compensation matrimoniale. La complexité des relations, impliquant selon les cas épouse, femme gagée ou esclave, ne se réduisait pas, comme le croyaient les Européens administrateurs ou ethnologues -, à l'achat par un homme de droits sur la femme. La circulation des femmes était d'autant plus développée qu'elle était liée à celle de la compensation matrimoniale, elle-même susceptible de circuler considérablement. Parfois, on l'a vu au début de cet article, la circulation des femmes était rendue aisée par celle de la dot: il suffisait qu'un nouvel époux remboursât le précédent mari pour que l'affaire soit faite, et ceci n'impliquait guère l'acceptation de la femme; le divorce se réglait entre les hommes et les familles, et non entre les deux partenaires. Souvent néanmoins, le départ d'une femme demeurait impensable pour le lignage du mari, ce qui donnait à l'adultère une tout autre signification que celle impliquée par le mariage religieux ; le mari était offensé par l'homme qui lui avait pris sa femme, et c'était celuici qui lui devait compensation. Là encore, la femme n'était pas impliquée ou peu dans les débats. En fait, les mots de divorce et d'adultère eux-mêmes donnent une idée fausse des relations.

On peut noter, à travers l'exemple des LoDagaa des Territoires du nord-ouest du Ghana, à quel point l'incompréhension était grande. Les termes traduits par mariage étaient, selon le genre, ceux de pog (prendre une femme) ou de kul sir (aller chez un homme) : il n'y avait pas sacrement, mais accord qui n'impliquait pas nécessairement un engagement permanent (Hawkins 2002). Ce que l'homme demandait à une femme, ce n'est pas un serment de fidélité, mais fondamentalement deux actions : de lui donner des enfants, et d'assurer la subsistance du groupe. Ainsi, quand un travailleur migrant était absent et que sa femme était partie vivre avec un autre, il s'agissait bien pour les gens du lieu d'une femme qui avait changé de mari, et non d'un adultère comme le comprenaient les tribunaux coloniaux. Que le nouveau mari argue comme raison « qu'il voulait épouser la femme » dépassait l'entendement de l'agent colonial, pour qui il était aberrant d'épouser une femme qui était déjà mariée. Soucieux de protéger les droits du migrant, le tribunal «coutumier» installé par les Britanniques, parfois appelé à se prononcer (souvent sur plainte de la femme), condamnait celle-ci à retourner chez «son mari", ce qui n'arrangeait personne: ni l'ancien mari qui aurait préféré récupérer sa mise, ni la femme qui avait fait sa vie (et des enfants) ailleurs, ni le nouveau mari qui n'entendait pas se défaire de la force de travail qu'il avait ainsi acquise. Ce n'est guère avant 1936 que les LoDagaa eurent, sur injonction de l'administration coloniale, à édicter une Native Customary Law sur le "mariage » et le « divorce » impliquant une procédure légale réglementant de façon rigide ce qui avait constitué auparavant un ensemble de pratiques sociales fluides, exclusivement régulé par la circulation de la dot.

Or celle-ci pouvait durer longtemps, faisant passer par des étapes successives ce que les observateurs occidentaux prirent pour des états distincts. Ce qui était vrai des LoDagaa l'était aussi, sous des formes un peu différentes, des Ashanti (Tashjian \& Allman 2002) : naguère, l'ethnologue Rattray (1929) avait différencié une série de types matrimoniaux distincts, dont les trois principaux étaient : le mariage entre un homme et une femme libres (adeyie awadie), le concubinage (awowa awadie), ou le mariage entre un homme libre et une femme gagée ou pawn (kuna awadie). La première catégorie méritait seule à ses yeux le qualificatif de mariage (au sens occidental du terme) : le versement de la dot (aseda) conférait aux époux des obligations réciproques, le plus important, selon 
l'anthropologue, étant le droit pour l'homme de réclamer un dédommagement financier en cas d'adultère de la femme. Dans le cas moins formel du concubinage (bien qu'il soulignât que, contrairement à l'Occident, il n'y avait pas de honte attachée à cette forme d'union), il insistait également sur les droits et obligations sexuels des époux, la seule différence étant que le mari n'avait pas à réclamer de dédommagement en cas de "faute» de la femme; dans le troisième cas, de femme gagée, celle-ci, donnée en mariage en échange d'une dette, était particulièrement dépendante de son mari. Or, la réalité démontre que le passage était fluctuant d'un état à l'autre d'union: ainsi, les époux vivant sous le mode awowa awadie signifiait simplement que le paiement de la dot n'était pas encore effectué ou inachevé, et cela pouvait prendre des années; car le processus comprenait plusieurs étapes: d'abord de petits présents à la famille et au père de la jeune femme ; puis à nouveau des cadeaux plus sérieux à la femme elle-même et à sa famille et, enfin, au terme d'un long périple, le versement final du «mouton de dieu » (anyame-dwan). Celui-ci signifiait la passation au mari de la protection spirituelle que la femme avait jusqu'alors, depuis sa naissance, reçue de son père. Mais ceci n'empêchait nullement que l'épouse ne pût devenir à son tour femme gagée : il suffisait pour cela que sa propre famille se trouve en difficulté, et ressente par exemple le besoin d'emprunter au mari: le créditeur se remboursait alors sur le travail de sa femme. Contrairement à ce que pensait Rattray, le fondement du mariage ne reposait pas sur l'exclusivité sexuelle du mari sur sa femme, mais sur un échange complexe d'obligations et de responsabilités réciproques de travail entre la famille du mari et celle de la femme qui pouvaient être constamment contrôlées et renégociées.

Les travaux de Gwendolyn Mikell sur les implications juridiques complexes du mariage, du divorce ou de l'héritage en pays de tradition matrilinéaire, en pays akan au Ghana, sont éclairants sur les adaptations en cours jusqu'à nos jours. En fait, l'affaire risque de devenir tragique en cas de veuvage de la femme, puisque les biens du mari, selon la coutume, vont aux enfants de ses sœurs. La confédération des chefs akan avait déjà proposé, en 1938 et surtout en 1948, que la veuve et ses enfants pussent hériter du tiers des biens du défunt. Mais les tribunaux ne suivirent pas, car le gouverneur britannique de l'époque n'avait pas contresigné la proposition. Même problème dans le cas, fréquent, où la femme a divorcé, ou bien si elle est laissée enceinte sans avoir été épousée dans les règles. La situation n'est guère meilleure en cas de polygamie, quand la femme s'est vue préférer une autre épouse plus jeune et que le mari n'habite plus sous le même toit. Jusqu'à il y a peu, même devenue de fait chef de famille monoparentale, la femme n'avait pas la possibilité légale d'exiger une aide de son mari.

Devant le blocage de la situation, les femmes ont, depuis décembre 1981, la possibilité de soumettre leurs problèmes familiaux à des tribunaux spécialisés dits Family Courts. La crise des années 1980 a précipité l'évolution. Alors qu'il n'était guère pensable, auparavant, de parler hors du lignage de difficultés domestiques, les procès se multiplient depuis quelques années. On les compte par milliers auprès des Greater Accra Family Courts depuis 1985, année où elles ont été explicitement chargées des questions touchant au mariage, au divorce, à l'héritage et aux legs, dans un contexte très majoritairement matrilinéaire. La plupart des plaintes émanant de femmes dont près de la moitié (42,5\%) travaillent dans le commerce informel et 20 \% sont au chômage, et dont un tiers environ ne sont pas mariées (et seulement $3 \%$ se sont mariées selon la loi ou Marriage Ordinance), sont dues à l'incapacité des femmes à obtenir une aide soit de leur lignage, soit de leur mari. Or le fait même de porter devant la justice une affaire conjugale, jusqu'alors considérée comme ne relevant que de la famille coutumière, 
suffit à faire perdre la face au mari. Les requêtes concernent l'entretien des enfants ; certaines réclament en plus une participation aux frais de scolarité ou une aide en cas de maladie (ce qui n'a rien d'étonnant compte tenu de l'augmentation du taux de mortalité infantile). D'autres, en revanche, refusent toute aide à laquelle elles auraient légalement droit, et notamment une pension alimentaire pour les enfants en cas de divorce, de peur que le mari n'argue de cette obligation pour revendiquer sur les enfants un droit que ne lui reconnaissent pas les coutumes matrilinéaires (Mikell 1997 : 96-1208). L'intervention de la justice va en effet beaucoup plus loin qu'une simple mesure sociale; elle transforme l'ensemble du système puisque, à partir du moment où l'homme participe à la charge des enfants, il revendique son droit à les contrôler : un acte tout à fait nouveau est, surtout chez les Fanti où la fonction paternelle est plus marquée qu'ailleurs, la revendication du droit de garde au bénéfice du père (et donc $\mathrm{du}$ lignage paternel). À l'opposé, puisque la reconnaissance d'une paternité risque d'occasionner des frais qu'ils ne sont pas prêts à assumer, beaucoup d'hommes répugnent à épouser la fille qu'ils ont engrossée et surtout refusent de reconnaître leur progéniture ; or cela s'inscrit à l'opposé du rite d'accueil consistant à "nommer " l'enfant, c'est-à-dire à le présenter officiellement comme membre de la famille, et porte donc à celui-ci un sérieux préjudice.

Enfin, la loi a des effets pervers : elle fait perdre à la mère ses prérogatives anciennes en faveur d'une conception occidentale qui tend à la faire reconnaitre comme dépendante de l'homme. Beaucoup de femmes sont défiantes envers ce changement de mentalité qui risque à terme, si les pères négocient leur prise de responsabilité, d'amoindrir leur droit à l'autonomie. D'autres, au contraire, se montrent prêtes à utiliser la possibilité qui leur est donnée de se démettre d'une partie de leurs obligations. Mais le cas n'est pas encore fréquent de cette femme akan qui demande le divorce parce que son mari veut lui imposer une co-épouse : elle a eu de lui trois enfants dont le dernier est un nourrisson de cinq mois seulement. Contre la volonté du juge qui considère normal que la femme continue d'allaiter le petit dernier, elle en refuse la garde, bébé sevré (ditelle) inclus :

«Il m'a menacée de prendre mes enfants : qu'il les prenne, mais tous. Il ne va pas me laisser un bébé qui fera que personne ne voudra m'épouser. [Et d'ajouter] Juge, je vous le répète ; je veux qu'il prenne tous les enfants » (ibid. : 115).

Souvent, la séparation est progressive, n'impliquant pas un « divorce » en bonne et due forme : la femme s'absente d'abord de plus en plus pour son commerce, ses affaires de famille et finalement s'installe ailleurs laissant la place à une autre. À noter que la tolérance est moindre chez les Wolof que chez les Éwé du Togo ou les Akan du Ghana : la première séparation est bien acceptée, mais une femme qui a des enfants d'un grand nombre de maris - trois ou davantage - est considérée comme volage et encourt la réprobation de l'entourage.

On voit la richesse potentielle de ces sources, y compris en ville les sources notariales. Celles-ci sont encore à peine exploitées du côté français par les chercheurs sauf en des cas bien précis, comme par exemple l'énorme thèse malheureusement non publiée de Roger Pasquier (1987) sur la société saint-louisienne au milieu du XIX ${ }^{e}$ siècle.

Femmes et droit foncier

37 Les auteurs s'accordent en général pour insister sur une réduction du droit des femmes sur la terre à l'occasion de la colonisation, toujours pour la même raison : la primauté du paternalisme juridique occidental. Ceci paraît patent en régime matrilinéaire où, 
traditionnellement, la femme recevait de sa propre famille un patrimoine qui demeurait son bien propre. Les profits qu'elle pouvait en tirer - soit qu'elle bénéficiât d'un lopin de terre, soit qu'elle ait reçu quelques têtes de bétail - lui permettaient notamment d'intervenir dans le choix d'une épouse pour son fils, puisqu'elle allait de ce fait participer au paiement de la compensation matrimoniale. Il est donc intéressant de dépister, dans les sources judiciaires, les plaintes pouvant émaner de ces femmes qui s'estimaient lésées, par exemple en cas de divorce ou de décès du mari.

Deux cas se présentent dans les archives judiciaires britanniques; ils correspondent grosso modo au système préexistant, selon qu'il était à prédominance matrilinéaire ou patrilinéaire. En fait, on ne trouve de recours féminin à la justice que dans le premier cas en zone rurale, ou bien lorsqu'un milieu social averti commence de réagir en milieu urbain ; en effet, si la famille du mari était prompte à chercher à profiter du nouveau climat aux dépens de la femme, les colons restaient parfois attentifs à préserver les " coutumes» ancestrales en faveur de celle-ci. Le cas a été notamment étudié par Kristin Mann (1991) chez les citadines yoruba de Lagos qui, au début du siècle, se battirent parfois bec et ongles pour garder le contrôle de leur propriété, en l'occurrence la maison souvent achetée avec leurs gains de commerçantes (marketwomen).

39 La pénétration du capitalisme marchand occidental a introduit à Lagos, au tournant du $\mathrm{xx}^{\mathrm{e}}$ siècle, de nouvelles formes de propriété et de droit foncier qui désavantageaient les femmes, même si le régime patrilinéaire était mâtiné de fortes influences matrilinéaires. Dès le milieu du XIX siècle - Lagos devint protectorat britannique en 1851 -, l'habitude fut prise, aussi bien par le chef de Lagos que par les autorités britanniques bientôt considérées comme le propriétaire éminent de l'ensemble, de concéder des lots en toute propriété. Le roi Dosunmu, qui régnait dans les années 1850, fit ainsi 72 dons de terre par écrit: seulement 4 allèrent à des femmes. Le gouvernement de la Couronne n'alla guère plus loin. Aucune femme n'accumula autant de biens que quelques propriétaires fonciers masculins. Par exemple, entre 1863 et 1871, un commerçant illettré, Taiwo Olowo, obtint 14 concessions de la Couronne dans les meilleurs quartiers de la ville. Entre 1869 et 1876, Sunmonu Animasaun, un négociant musulman prospère d'origine servile, obtint à son tour 7 lots ; entre 1868 et 1893, il acquit par achat ou échange 30 autres lopins supplémentaires. Si l'on compare avec des femmes fortunées, on constate que Fanny Barber et Rebecca Phillips Johnson, deux des plus riches femmes de Lagos, toutes deux sierra-léonaises éduquées (anciennes esclaves libérées et rapatriées) ne possédaient respectivement que 6 et 9 lots, soit beaucoup moins que ces deux marchands locaux analphabètes.

Il n'empêche qu'officiellement les femmes n'étaient pas exclues de la propriété foncière urbaine ; plusieurs fois, elles eurent devant la justice gain de cause face aux tentatives familiales de remettre la main sur leurs biens propres au nom de la loi coutumière ou de l'autorité du mari. Ainsi, en 1870, une femme dénommée Rokosi avait acheté un lopin. L'année suivante, elle quitta Lagos après avoir confié l'acte de concession de la Couronne à sa sœur. Celle-ci fut circonvenue par un parent, Moses Johnson, qui, mis en prison pour dette, se fit donner le papier pour obtenir un prêt. En 1887, la société prêteuse se préparait à mettre la maison gagée en vente. Rokosi engagea un procès et le gagna, ayant réussi à convaincre le juge que sa sœur avait agi sans son autorisation. Une autre marchande, Mary Macaulay, fit procès à un négociant et à un usurier pour récupérer sa maison : en 1874, elle avait épousé son mari à l'Église anglicane. Trois ans 
plus tard elle hérita d'une maison de sa tante paternelle. Au début des années 1890, son mari, qui avait besoin d'argent, prit l'acte foncier et hypothéqua la maison pour obtenir un prêt sans en informer sa femme. La maison fut vendue trois ans plus tard. Quand Mary s'en aperçut, elle alla en justice et rompit avec son mari. Là encore, la cour lui donna raison et la rétablit dans son droit.

En somme, les femmes devaient en permanence veiller à se défendre et ne trouvaient pas toujours une oreille aussi bienveillante. On relève ainsi dans les archives judiciaires des cas exemplaires: Aina avait vécu avec son mari Idowu pendant 22 ans. Quand il mourut, le jeune frère réclama, selon la coutume, à la fois la maison et la femme. Celleci s'était sauvée en emportant l'acte de propriété. Son beau-frère déposa un recours à la Cour Suprême qui lui donna raison: la femme dut rendre l'acte et, pour garder la maison, se résigner au lévirat. Les archives missionnaires regorgent de cas où les femmes recouraient aux services de la mission pour défendre des droits bafoués.

Le marché de la terre se développa de plus en plus au début du $\mathrm{xx}^{\mathrm{e}}$ siècle, et les femmes eurent beaucoup de mal à s'y maintenir, pour plusieurs raisons. Leur accès au capital était limité par leur difficulté à obtenir des prêts personnels. Quand une femme, Asiatu, demanda en 1882 au marchand africain Charles Foresythe de lui vendre ses marchandises à crédit, il refusa à moins qu'elle ne pût garantir l'avance sur un bien hypothéqué. Or, à la différence des hommes, les femmes n'obtenaient guère l'autorisation nécessaire de la famille sur des biens généralement en indivision : aucune n'était capable de faire ce que fit Taiwo Olowo, un riche marchand qui, en 1867, hypothéqua 17 propriétés pour obtenir un prêt qui lui permit d'acquérir 40000 gallons d'huile de palme. Cinq ans plus tard, J. P. L. Davies hypothéquait 14 lopins pour jouir d'un crédit annuel de $60000 £$. En 1919, P. J. C. Thomas hypothéquait à son tour ses propriétés contre un crédit de $150000 \mathrm{E}$. Les affaires commerciales des femmes, disposant de moins de crédit, n'avaient pas, sauf exception, cette envergure, et ne leur permettaient ni de s'agrandir ni d'investir dans le foncier, car le commerce était aussi exigeant en main-d'œuvre: il fallait du personnel pour transporter, stocker, vendre, etc.; les hommes utilisaient la hiérarchie inhérente à la maisonnée : leurs épouses, leurs enfants, leurs dépendants, leurs esclaves. Les femmes ne disposaient que d'elles-mêmes. Elles devaient, pour réussir, s'installer à leur propre compte, c'est-à-dire devenir chefs de famille indépendantes. Quelques-unes le firent dès la fin du XIX siècle, profitant de l'avantage que leur offrait la pénurie relative de femmes provoquée par l'arrivée massive d'hommes de l'intérieur, ce qui leur permettait de changer de mari si celui-ci s'y opposait (Mann 1991 : 702).

En situation de patrilinéarité affirmée, qui ne coïncidait que trop bien avec l'esprit victorien du temps, la femme n'avait quasi aucune chance de trouver gain de cause. Au Lesotho, par exemple, la subordination des femmes a précédé la colonisation, mais l'intrusion du capitalisme a perpétué et intensifié cette dépendance : le tabou exercé sur les femmes qui n'avaient pas le droit de posséder des têtes de bétail les excluait de la richesse principale de la région; les seules femmes qui purent atteindre quelque aisance étaient les veuves, autorisées à jouir des biens de la maisonnée jusqu'à leur mort ; mais ceux-ci passaient ensuite aux héritiers mâles exclusivement (Elredge 1991 : 729). En Rhodésie du Sud (Zimbabwe), les femmes eurent la malchance de voir le droit coutumier "fixé» avec l'aide d'un juriste britannique redoutable, H. Child, dont l'ouvrage fit autorité auprès des tribunaux coutumiers jusqu'à l'indépendance de 1980 et même au-delà. Les principales sociétés du Zimbabwe, shona aussi bien que ndebele, 
étaient de tradition patrilinéaire. Mais il n'existe guère de preuve que les femmes aient été autrefois privées du droit de posséder au point où le défini H. Child. Les femmes shona avaient pu jouir de deux types de biens : umai (biens de maternité), c'est-à-dire la part en têtes de bétails qui revenait à la mère en cas de mariage d'une de ses filles, et mavoko (biens de ses mains). L'umai pouvait consister en une vache ou/et une chèvre, donnée par le gendre à sa belle-mère, notamment lors d'une grossesse de sa fille. Le cadeau incluait la portée de la bête, c'est-à-dire la possibilité pour la femme d'accroître son bien. La femme conservait le plein contrôle de son petit troupeau qui, en cas de décès, revenait à son propre lignage. Le mavoko relevait de son travail personnel, par exemple d'un artisanat en poterie, de sa fonction de matrone ou d'herbaliste. Une production communément pratiquée par les femmes était la brasserie artisanale de bière. Cela dit, une femme qui pour une raison ou une autre retournait chez ses parents emportait avec elle peu de choses, sinon ses pots et ses pagnes (May 1983:66).

Or, à la suite de la loi «coutumière " régulée par les Britanniques en accord avec des chefs soucieux de renforcer leur autorité, Child reconnut bien le droit des femmes à posséder quelques biens en nature (dont des têtes de bétail). Chez les femmes tonga, de tradition matrilinéaire, il reconnut quelques autres droits, tel celui de posséder des biens en propre par héritage, dot, réparation ou achat. Les femmes étaient propriétaires de la récolte produite dans les champs qu'elles possédaient en propre, et il apparaît même qu'avant la colonisation elles pouvaient posséder de la terre. Cela dit, à partir du moment où fut introduite la monétarisation, les femmes se virent refuser le droit de posséder de l'argent. Child (1976 : 91) l'énonce clairement :

"Si une femme va travailler à l'extérieur avec l'accord de son mari et sous son contrôle, tout l'argent qu'elle gagne lui appartient à lui, qu'il s'agisse du pays Shona ou Ndebele [...] si une femme Shona ou Ndebele a été autorisée à garder ses gains, la propriété acquise avec son propre argent peut rester son bien sa vie durant en qualité de mavoko. »

Bien qu'apparemment sans fondement réel, cette règle, en vertu de l'autorité de Child, demeura longtemps invoquée par les cours de justice. On la trouve encore justifier un jugement de 1969:

«Malheureusement pour la plaignante, bien que quelques Africaines aient pu acquérir une certaine émancipation [...] en vertu de leur mariage chrétien, la loi foncière est telle que, en dépit de la solennité d'un tel mariage, la propriété des époux doit demeurer telle qu'elle est prévue par la loi et la coutume africaines » (ibid. : 67).

Le résultat était que, en cas de divorce, la femme perdait tout ce qu'elle avait investi dans la maisonnée conjugale, que ce fût en biens ou en épargne. On comprend dans ces conditions l'indignation des infirmières de Hararé qui, au lendemain de l'indépendance de 1980, réclamèrent une modification de la loi pour disposer de leur salaire : car on voyait encore à la sortie de l'hôpital, le jour de la paie, leur mari faire la queue pour le toucher à leur place'.

La propriété foncière (au-delà des réserves) fut longtemps interdite aux hommes par le gouvernement rhodésien ségrégationniste. Néanmoins, à partir des années 1940, face aux revendications des Africains, la vente d'un quota de $8 \%$ des terres leur fut réservée ; mais c'est aux hommes que la loi de 1951 prévit d'ouvrir, à titre individuel, le marché de la terre (cette ouverture fut d'ailleurs supprimée quand le Front nationaliste blanc prit le pouvoir en 1962). La femme n'était admise comme chef de maisonnée que si elle était « célibataire, veuve, divorcée ou officiellement séparée » - c'est-à-dire 
si aucun homme n'était présent, même à titre temporaire. Au plus, $10 \%$ des femmes entraient alors dans cette catégorie. Depuis l'indépendance, malgré un vaste programme de recolonisation africaine des terres en 1982 et alors que les femmes indépendantes ne cessent d'augmenter, le gouvernement poursuivit dans le même esprit en définissant la priorité des "groupements familiaux"-ce qui favorise évidemment les chefs de famille de sexe masculin. Une déclaration de $1981 \mathrm{du}$ secrétaire d'État à la Terre (Deputy Minister of Lands) est à cet égard exemplaire :

«Nous ne pouvons attribuer des terres aux hommes pourvus d'emploi puisqu'ils n'ont pas le temps de la travailler [...]. Pour l'instant ils ont déjà des terres considérables cultivées par des personnes sans emploi [c'est-à-dire leurs épouses !] » (Jacobs 1984 : 33-49).

48 C'est que les femmes mariées accomplissaient toujours la quasi-totalité du travail agricole. Mais elles ne sont même plus protégées par les usages coutumiers qui chargeaient l'homme d'assurer la subsistance de ses épouses et de leur progéniture. Avec la fin du lévirat, le sort des veuves est particulièrement fragile : elles constituent avec les divorcées la majeure partie des quelque $10 \%$ de paysans sans terre vivant à la campagne dans la plus extrême pauvreté.

Avec la création de l'État zimbabwéen, les femmes ont réclamé avec énergie la réforme de leur statut qui avait été «oubliée » à l'indépendance. Mais elles sont loin d'être vraiment émancipées, en vertu d'un « discours moral» dont l'héritage colonial n'est pas encore dissipé (Jeater 1993).

50 Cette attitude face aux droits des femmes sur la terre n'est pas spécifique à l'Afrique australe. On la retrouve peu ou prou partout; la situation des femmes s'aggrave au fur et à mesure que s'affirment à la fois le manque de terre et la montée d'une bourgeoisie agraire essentiellement masculine.

51 Ainsi, au Kenya où les femmes contribuent toujours à plus de $80 \%$ de la production agricole, le droit à la propriété des femmes du Kenya occidental, luo, gusii et luyia, fut très limité. En vertu du patrilignage, elles n'héritaient pas de leur père au bénéfice de leurs frères. Comme en Rhodésie, elles ne se voyaient reconnaître la jouissance de la terre, du bétail ou de tout autre bien que par l'intermédiaire de leur mari. Hors mariage - qui était conçu pour la vie, avec peu de possibilités de divorce et la coutume du lévirat en cas de veuvage -, elles n'avaient d'autre solution que de se réfugier à la mission ou en ville - ce qui rend compte, d'ailleurs, de la précocité de leur incitation à migrer. Si elles arrivaient à accumuler un petit pécule, à partir des quelques têtes de bétail qui leur étaient données ou de leur activité artisanale de nattes ou de poteries, le profit qu'elles pouvaient en tirer était vulnérable puisque toute accumulation de richesse revenait de droit au mari, seul gérant légal des biens de la famille. Tout cela fut encore accentué quand les autorités, dans les années 1950, entreprirent de codifier la loi coutumière: les chefs luo eux-mêmes reconnurent un peu plus tard qu'ils avaient «réécrit en partie la coutume pour remettre au pas les jeunes femmes». Ils s'opposèrent, en particulier, durablement à l'enregistrement de propriétés individuelles qui aurait risqué de favoriser les femmes, chefs d'exploitation de fait en l'absence des maris partis travailler au loin (Hay 1982).

Aujourd'hui, les Kenyanes sont responsables d'un quart des exploitations - il est vrai les plus petites et les plus pauvres car c'est là que la migration urbaine des hommes est la plus élevée; elles ne possèdent, en contrepartie, que $5 \%$ des terres sur les 7,6 millions d'ha enregistrés en 1978 en propriété individuelle. Pourtant, dès 1966 le gouvernement 
avait suscité un "Programme de groupes de femmes", destiné à améliorer leur "participation au développement »; le Plan de 1977 proposait un effort particulier en direction des agricultrices chefs de famille tout en n'en spécifiant guère les attendus. Or, en 1984, un responsable local n'en déclarait pas moins, lors d'une séance de préparation à la conférence sur les femmes organisée l'année suivante à Nairobi par les Nations Unies :

«Bien que les femmes n'aient pas accès aux titres fonciers sur la terre familiale, elles participent largement aux décisions concernant leur exploitation. Nous ne devons donc pas perdre de temps à envisager l'accès des femmes à la propriété comme un moyen d'augmenter la production » (Davison 1987).

Une femme kikuyu, qui fournit la quasi-totalité de la subsistance de la maisonnée, répond à cela : «Sans la terre, nous ne sommes rien. » En cette région patrilinéaire, les femmes cultivaient jadis jusqu'à dix parcelles différentes auxquelles elles accédaient par mariage. Compte tenu de la pression rurale, elles n'en ont plus aujourd'hui que deux ou trois dont la taille s'est fortement réduite. Le plus grave est que les femmes ne peuvent recourir au crédit agricole pour améliorer leur équipement ou acheter des terres, car celui-ci exige justement d'être garanti en terres dont la propriété relève de leur mari. Quant à la femme célibataire ou divorcée, elle demeure ou revient chez son père qui lui confie une parcelle en attendant de la marier, mais répugne à la lui donner pour ne pas diminuer la part des fils. Plus tard elle la recevra, de la même façon, de l'un de ses frères puis de ses enfants. Si l'homme dont elle dépend travaille ailleurs, et s'il veut par exemple vendre un lopin pour se faire construire une maison en ville, elle reste sans défense face à la perte de son gagne-pain. Or, le respect et le devoir dus par les fils à leur mère tendent à s'effriter face à l'expansion du marché foncier... Récemment, les femmes ont commencé à s'organiser en coopératives pour rassembler le capital nécessaire à l'achat du matériel ou de la terre devenus nécessaires à leur subsistance. Mais, dans les années 1990, $11 \%$ seulement d'entre elles faisaient partie des « groupes de femmes » encouragés par le gouvernement. Le plus souvent, il ne leur restait qu'à migrer à leur tour vers la ville.

Le cas le plus tragique était celui des veuves, car fréquent était le pays dont les " coutumes », figées par la colonisation, ne reconnaissaient d'autre droit à la femme que le lévirat. La veuve n'avait en effet le droit - en régime patrilinéaire où cela revient aux fils, comme en régime matrilinéaire où les biens vont aux enfants de la sœur utérine - ni d'hériter ou d'administrer les biens de son mari, ni même de l'enterrer. Ce n'est que récemment que les États se sont efforcés de réparer cette injustice ${ }^{10}$. Auparavant, notamment en Zambie, même si la loi coutumière différenciait le droit d'héritage de celui de l'administration des biens du défunt, la veuve n'avait droit ni à l'un ni à l'autre. Les cours de justice et l'administration coloniale se sont efforcées de remédier à cet état de fait, surtout en zone urbaine où la gestion des biens n'était guère laissée sans contrôle à la seule communauté familiale. Sur requête de la veuve récusant la loi coutumière, la cour pouvait faire intervenir un fonctionnaire spécialisé, l'administrateur général, chargé de régler la succession. En général, il prélevait une part de l'héritage en faveur des enfants du défunt (environ $50 \%$ ) et de la veuve (de l'ordre de $25 \%$ ). Dans le reste du pays, les cours locales pouvaient aussi moduler la loi coutumière « de sorte qu'elles n'apparaissent pas contraires à la justice et à la morale naturelles $»^{11}$. Mais elles étaient composées d'hommes âgés et peu éduqués, choisis en raison de leur compétence en matière de coutumes. Dès lors, rares furent les veuves désignées comme administratrices de leurs biens. D'abord parce que très peu de 
femmes en firent la demande, soit qu'elles ne fussent pas informées de leurs droits, soit que la chose apparût si contraire aux usages qu'elle restait hors de leur portée. Très peu de femmes s'opposèrent à la nomination de l'administrateur proposé par la famille. Tout cela fut en principe aboli en 1989, quand une nouvelle répartition des biens du mort fut édictée ${ }^{12}$.

Le sort des femmes en régime patrilinéaire n'est pas meilleur en Afrique occidentale : face à la crise foncière que subit le sud Bénin en raison de la croissance démographique rapide, la discrimination foncière est patente en défaveur de la femme au moment de l'héritage. Les femmes sont obligées d'avoir recours à des solutions alternatives, comme le métayage, la donation entre vifs (le père donne des parcelles à ses filles de son vivant) ou l'emprunt de parcelles au sein de la famille... (Sohinto $2001: 67$ ).

On doit signaler le cas particulier de la restriction du droit des femmes sur la terre édictée par l'émir de Kano, dans le cadre du gouvernement indirect (Pierce 2003). Un ouvrage récent (Pierce 2006) démontre que même au Nigeria du Nord, supposé protégé des déformations coloniales par le "gouvernement indirect " qui aurait respecté les droits de la terre coutumiers antérieurs, les contresens sur les droits fonciers furent nombreux et persistants. Ils constituent à eux seuls une explication cohérente de beaucoup des difficultés héritées jusqu'à nos jours. On se trouve, à la différence des cas précédents, en terre d'islam gérée par la sharîa où, en principe, les filles ont droit à une demi-part. Et pourtant, en 1923, l'émir Usman prit l'étonnante décision d'interdire aux femmes d'hériter une maison ; il y ajouta, six mois plus tard, l'interdiction d'hériter une ferme. Ces biens devaient être partagés uniquement entre les fils et, en cas d'absence de lignée mâle, vendus. On retrouve chez l'émir et à sa cour la conjonction des arguments qui jouèrent aussi ailleurs pour limiter le droit des femmes. Le prétexte utilisé auprès des autorités britanniques fut l'argument traditionnel: les femmes n'auraient pas eu de droit sur la terre dans le droit coutumier patrilinéaire préalable à la jihad peule; le droit musulman des filles à l'héritage n'aurait commencé à être appliqué que sous l'émir Abdullahi (1855-1882). Les sources judiciaires du XIX siècle ne sont pas claires à ce sujet; les archives du conseil de justice de l'émir Abbas en 1913-1914 démontrent tout au plus la fragilité du droit des femmes, prompt à être battu en brèche par la parenté mâle, le chef du village, ou tout autre individu du sexe masculin (Christelow 1994). En fait, vrai ou faux, l'argument n'avait pas plus de valeur que celui utilisé par Child en Rhodésie. Mais il révèle un objectif similaire, celui de limiter au maximum l'autonomie des femmes. Toute femme indépendante était une karuwanci, mot traduit par "prostituée » ou, au mieux, «femme entretenue », c'est-àdire une menace directe contre l'ordre social dominant; d'où le rejet de toute indépendance féminine par rapport au pouvoir masculin. Une femme chef d'entreprise était considérée comme impensable. La femme était condamnée à ne vivre que sous la dépendance de son père, de son mari ou de ses fils. La mesure ne fut révoquée qu'en 1954 par le nouvel émir. Le motif officiel fut celui de la pauvreté des vieilles femmes tombées dans la misère et délaissées par leur progéniture. Il est probable que l'évolution générale des mœurs n'y fut pas non plus étrangère.

Car, depuis les Indépendances, surtout depuis les années 1990, la prise de conscience de leurs droits par les femmes se développe rapidement. Nul doute qu'elle rend de plus en plus fréquent, comme Gwendolyn Mikell (1995) et Takyiwaa Manuh (1997 : 77-95) l'ont étudié pour le Ghana, le recours des femmes à une justice qui, bon gré mal gré, s'adapte à leurs revendications. Il apparaît important de reconstituer cette évolution à travers 
les lois quand elles ont été votées, et en tout état de cause à travers les recueils de jurisprudence qui témoignent de l'évolution des mœurs par les modifications dans l'interprétation de la loi, "coutumière " ou non, par les tribunaux sollicités. Ainsi s'ouvre un terrain vaste et prometteur aux nouvelles générations de chercheurs en histoire et sciences sociales.

\section{BIBLIOGRAPHIE}

\section{NOTE SUR LES SOURCES DE LANGUE FRANÇAISE}

Les inventaires dressés par les archivistes du Sénégal, dans les archives de l'ex-AOF de la série M (actes et tribunaux judiciaires) et de la série $4 \mathrm{Z}$ (actes notariés) sont des instruments de travail précieux. Reste à les exploiter au mieux en ce qui concerne la situation des femmes. On remarque, au niveau des index, aussi bien pour les actes notariés que pour ceux de justice, la très faible fréquence - à la différence de ce qui se produit pour les actes de naissance ou de mariage de la mention de divorces, sauf à la fin du XIX ${ }^{\mathrm{e}}$ siècle (année 1891 seulement pour les actes de divorce des communes de Gorée et de Dakar, dossiers 5M/543, 544, \& 546 ; années 1921-1923 seulement pour la justice indigène, dossier $6 \mathrm{M} / 316)$. Est-ce à dire que l'on ne divorçait plus à Dakar depuis la fin du XIX ${ }^{\mathrm{e}}$ siècle ? Certainement pas. Il est possible que, l'islamisation progressant, beaucoup d'actes de répudiation ne soient guère passés par la justice à la française, même « indigène "; il est plus probable que de nombreux actes de ce genre sont perdus dans la masse des dossiers dont le contenu hétérogène donne lieu à une énumération aussi lourde qu'approximative : seul un examen attentif de ces documents d'archives peut en extraire la substantifique moelle, comme le fait Awa Yade dans le présent numéro. En ce qui concerne la transmission des biens, par les femmes ou non, les archives notariales constituent une richesse extraordinaire, et il est remarquable de les trouver réunies sur une aussi longue durée au moins pour les Originaires des Communes, même si l'inventaire s'arrête aux années 1920 : ce qui ne peut qu'encourager à rechercher et répertorier la suite... C'est à ce titre que les six répertoires concernés sont riches de promesses : et pour commencer, outre celles déjà soutenues à Dakar, que de maîtrises en perspective !

FONDS FRANCOPHONES À INVENTORIER

- Archives notariées de Saint-Louis, du Sénégal et Dépendances, sous- série 4Z2 : 1786-1924, Dakar, 2001, 46 p.

- Actes notariés de Gorée-Dakar (4Z1) : 1819-1919, et Archives des Particuliers (1Z) : 1787-1987, Dakar, 2001, 79 p.

- Tribunaux judiciaires 1819-1956 du fonds de l'AOF, Série M, Dakar, 1996, 158 p. (avec une utile bibliographie de base).

- Justice indigène (Sénégal) 1838-1954, sous-série 6M, Dakar, 1996, 71 p.

- Actes judiciaires (Sénégal et Dépendances), 1824-1900, sous-série 7M, Dakar, 2002, 37 p.

- Sécurité publique au Sénégal, Police, Gendarmerie, Prisons (1840-1956), Série F, Dakar, 1997, 80

p.

RECUEILS DE JURISPRUDENCE 
- Recueil de jurisprudence AOF : cour d'appel (PO II $\left.8^{\circ} 16\right)$.

- Recueil de jurisprudence de l'Afrique noire : cour d'appel de l'AOF (PO II $8^{\circ} 16$ ).

\section{NOTES}

*. Un premier papier sur ce thème, remanié et complété depuis, avait été proposé au colloque international Archives judiciaires. Sciences sociales et Démocratie, Dakar, 14-18 décembre 2003.

1. Deux gros ouvrages collectifs font le point sur le droit contemporain et hérité concernant les femmes en Afrique (BOWMAN \& KUENYEHIA 2003), et plus spécifiquement sur le droit foncier et les femmes dans les pays suivants : Cameroun, Éthiopie, Mozambique, Nigeria, Rwanda, Sénégal, Ouganda (WANYEKI 1997, 2003).

2. Ainsi, jusqu'à la Seconde Guerre mondiale incluse, le droit français imposait à la veuve un tuteur pour ses propres enfants. La mère ne devenait co-tutrice qu'en cas de remariage.

3. Native Commissioner of Ndanga to Superintendent of Natives, Fort Victoria, May 18, 1914 (SCHMIDT 1991 : 743).

4. Ibid., 12 mai 1914.

5. CHANOCK, in HAY \& WRIGHT (1982 : XIII), citant la loi sur le mariage, édictée en 1971 en Tanzanie, loi qui ne faisait que reproduire un texte colonial de 1968 de l'ancienne fédération, qui n'avait depuis guère été appliqué en Afrique orientale, et pas du tout au Kenya.

6. Voir, sur la Côte-d'Ivoire (Toungara 1997), Libreville (RICH 2003) et le « Soudan » (ROBERTS 2005).

7. «Sexual Offences Act : a White Elephant? », The Herald (Zimbabwe), Thursday 24 July 2003, p. 16.

8. «Please for domestic relief : akan woman and family courts. »

9. Voir, pour un résumé de l'évolution des droits fonciers, à la campagne et à la ville, des femmes africaines, C. COQUERY-VIDROVITCH (1994:111-120).

10. Ces Intestate Succession Acts ont des dates variables : au Malawi dès 1967, mais au Ghana en 1985, au Kenya en 1972 mais en fait 1987, en Zambie en 1989 (HIMONGA 1993).

11. Règle 6 du Local Courts Act (HimONGA 1993 : 174).

12. $20 \%$ à la veuve, $50 \%$ aux enfants, $20 \%$ aux parents, et $10 \%$ au reste de la famille (HIMONGA 1993 : 188).

\section{RÉSUMÉS}

L'article dresse le bilan des recherches sur l'histoire genrée du droit. La justice concernant les femmes est désormais bien étudiée en Afrique anglophone, elle l'a été moins en Afrique francophone, d'où l'intérêt de procéder à la confrontation des recherches sur l'ensemble du continent, tout en cherchant à remédier à cette dissymétrie. L'objectif est de préciser évolution et confrontation des droits de la femme. 
En droit privé, on trouve une masse d'informations dans les "Grands Coutumiers " (côté français) et dans les recueils de jurisprudence puis dans les codes (codes de la famille par exemple); à cela s'ajoute tout ce qui a donné lieu à procès : archives juridiques, jurisprudence depuis le début de la colonisation, recours en grâce.

Trois domaines ont été privilégiés jusqu'à présent : les cas de sorcellerie, et surtout le régime du mariage et tout ce qui en dépend (y compris la sharîa) : polygamie, divorce, relations extra maritales, adultère, héritage ; le rôle de la dot nourrit le thème sur les droits fonciers et le droit au travail, depuis le droit d'autrefois (ancien) jusqu'au droit moderne, en passant par le droit coutumier figé par les colonisateurs et le droit colonial stricto sensu.

De ces analyses ressort le constat de ce que les femmes ont su faire d'un droit qui leur était globalement plutôtdéfavorable et comment il a pu être utilisé et manipulé au fil du temps.

Gender and Justice. Advanced Research in English Language. - The paper investigates the amount of knowledge accumulated for a gendered history of the law. Women's law and justice are more studied in English than in French. Therefore it is useful to compare research all over the continent so as to correct this francophone deficiency and to confront diverse evolutions.

The so-called "Grands Coutumiers" collected by the French colonial officers in the 1930s, collections of court judgments in English and, later on, Family codes are useful sources on private law. Court cases are extremely informative, collected in archives and in jurisprudence collections.

Three major themes appear: women's witchcraft; above all everything dealing with marriage (including the shari'a rules): polygamy, divorce, extra-marital relations, adultery, heritage. The brideprice question (lobolo) plays a major part to understand land laws and labour laws. One can follow the evolution of women's rights from precolonial times to the present, passing through colonial customary laws and western colonial laws.

A conclusion is that women were keen to distort rules that rather limited their rights. Women knew how to use and manipulate laws so as to change their legal conditions over time.

\section{INDEX}

Mots-clés : femmes, droit, genre, Grands Coutumiers, histoire du droit, langue anglaise, recherches

Keywords : women, law, justice, gender, research, évolution, history of the law, english language

\section{AUTEUR}

\section{CATHERINE COQUERY-VIDROVITCH}

Université Paris-VII/CNRS. 